\title{
Role of random biopsies in surveillance of dysplasia in ulcerative colitis patients with high risk of colorectal
}

\section{cancer}

\author{
Sawan Bopanna ${ }^{1}$, Maitreyee Roy ${ }^{2}$, Prasenjit Das ${ }^{2}$, S Dattagupta ${ }^{2}$, V Sreenivas ${ }^{3}$, V Pratap Mouli ${ }^{1}$, \\ Saurabh Kedia ${ }^{1}$, Rajan Dhingra ${ }^{1}$, Rajesh Pradhan ${ }^{1}$, N Suraj Kumar ${ }^{1}$, Dawesh P Yadav ${ }^{1}$, Govind Makharia ${ }^{1}$, \\ Vineet Ahuja ${ }^{1}$ \\ Departments of ${ }^{1}$ Gastroenterology, ${ }^{2}$ Pathology, and ${ }^{3}$ Biostatistics, All India Institute of Medical Sciences, New Delhi, India
}

Background/Aims: Recent data suggest that the incidence of ulcerative colitis (UC) related colorectal cancer (CRC) in India is similar to that of West. The optimum method for surveillance is still a debate. Surveillance with random biopsies has been the standard of care, but is a tedious process. We therefore undertook this study to assess the yield of random biopsy in dysplasia surveillance. Methods: Between March 2014 and July 2015, patients of UC attending the Inflammatory Bowel Disease clinic at the All India Institute of Medical Sciences with high risk factors for CRC like duration of disease $>15$ years and pancolitis, family history of CRC, primary sclerosing cholangitis underwent surveillance colonoscopy for dysplasia. Four quadrant random biopsies at $10 \mathrm{~cm}$ intervals were taken (33 biopsies). Two pathologists examined specimens for dysplasia, and the yield of dysplasia was calculated. Results: Twenty-eight patients were included. Twenty-six of these had pancolitis with a duration of disease greater than 15 years, and two patients had associated primary sclerosing cholangis. No patient had a family history of CRC. The mean age at onset of disease was $28.89 \pm 8.73$ years and the duration of disease was $19.00 \pm 8.78$ years. Eighteen patients (64.28\%) were males. A total of 924 biopsies were taken. None of the biopsies revealed any evidence of dysplasia, and 7/924 (0.7\%) were indefinite for dysplasia. Conclusions: Random biopsy for surveillance in longstanding extensive colitis has a low yield for dysplasia and does not suffice for screening. Newer techniques such as chromoendoscopy-guided biopsies need greater adoption. (Intest Res 2016;14:264-269)

Key Words: Colitis, ulcerative; Random biopsy; Surveillance; Dysplasia

\section{INTRODUCTION}

Ulcerative colitis (UC) and the burden of IBD are increasing in India, similar to other countries in the Asia-Pacific region. ${ }^{1,2}$ With the advent of effective therapies such as biologi-

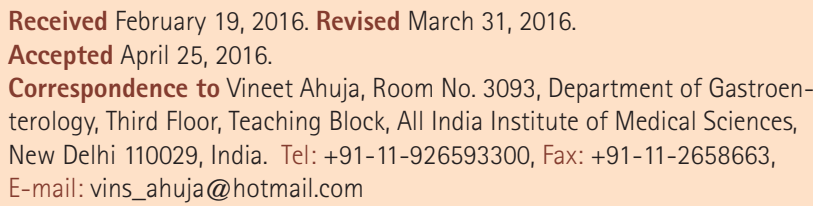

Financial support: None. Conflict of interest: None. cals and thiopurines, more patients are now able to maintain remission and have a longer colectomy-free survival. With increasing disease prevalence, the long-term complications of IBD are increasingly seen by physicians treating these diseases. The risk of developing colorectal cancer (CRC) is increased in patients with UC, which is one of the gravest long-term complications. In a meta-analysis by Eaden et $\mathrm{al}^{3}{ }^{3}$ the overall prevalence of CRC in UC was estimated as $3.7 \%$, with an overall annual incidence of $0.3 \%$. The cumulative risk of CRC was $8.3 \%$ at 20 years and $18.4 \%$ at 30 years. The risk of developing CRC in Indian patients is similar to that in patients in the West, as indicated by recent studies by Desai

\footnotetext{
๑ Copyright 2016. Korean Association for the Study of Intestinal Diseases. All rights reserved.

This is an Open Access article distributed under the terms of the Creative Commons Attribution Non-Commercial License (http://creativecommons.org/licenses/by-nc/4.0)

which permits unrestricted non-commercial use, distribution, and reproduction in any medium, provided the original work is properly cited.
} 
et al. ${ }^{4}$ Thus, even though the risk of sporadic CRC is low in India, the risk of CRC in our patients with UC is high and similar to that in patients in the West.

CRC is preceded by dysplasia, and surveillance strategies have been recommended to detect these pre-neoplastic lesions. The goal of endoscopic surveillance is to reduce the morbidity and mortality of colitis-associated carcinoma by either detecting and resecting dysplasia or detecting CRC at earlier, potentially curable stages. A cohort study of 149 patients ${ }^{5}$ with IBD-associated CRC found a $100 \%$ five-year survival of patients who were enrolled in a surveillance program compared with $74 \%$ in the non-surveillance group $(P=0.042)$. Of 30 patients with CRC-related deaths, only one patient died in the surveillance group compared with 29 in the non-surveillance group during the study period.

Thus, various guidelines endorse regular surveillance colonoscopies in high risk patients with UC. ${ }^{6,7}$ However, the ideal method of surveillance is still a matter of debate. Random biopsy sampling from different parts of the colon has been the traditional method for surveillance of dysplasia. It has been estimated that at least 33 biopsies per patient are needed to achieve a $90 \%$ confidence to detect dysplasia, if it is present. ${ }^{8}$ However, this method of surveillance is cumbersome to both the endoscopist and the pathologist, and is not costeffective. More recently, chromoendoscopy using various dyes has been recommended, followed by targeted biopsies from the area of mucosal abnormality. Chromoendoscopyguided targeted biopsies have been shown to have a higher dysplasia detection rate than random biopsies in various studies. $^{9-11}$ Thus, it is now recommended that when possible, chromoendoscopy should be used for dysplasia screening.

Chromoendoscopy is a recent technique and has a considerable learning curve. Not many gastroenterologists are well versed with the technique, and therefore the detection of mucosal irregularities may be difficult for a novice. The various guidelines recognize these limitations and add that when chromoendoscopy is not available, the traditional way (random biopsy sampling) should be used. In India, chromoendoscopy is only recently gaining popularity among practitioners and is not available everywhere. We therefore undertook this study to explore the yield of dysplasia in highrisk patients and whether random biopsies would suffice for screening.

\section{METHODS}

In this prospective study, we included patients with UC who were registered at the Inflammatory Bowel Disease clinic at the All India Institute of Medical Sciences, New Delhi. Ethical clearance for this study was obtained from the institution's ethics committee.

\section{Surveillance ileocolonoscopy}

Ileocolonoscopy was performed when disease activity was mild or in remission. Disease activity was assessed using the Simple Clinical Colitis Activity Index (SCCAI). Remission was defined as a SCCAI score of $<4$ and mild disease activity as a SCCAI score of $<7 .^{12}$ A polyethylene glycol electrolyte lavage solution was used in all patients for bowel preparation before ileocolonoscopy. We performed surveillance for dysplasia in those who had a high risk for development of malignancy. High risk factors considered for this were disease duration greater than 15 years, pancolitis, family history of colitis carcinoma detected at age $<50$ years, or co-existing primary sclerosing cholangitis (PSC). Ileocolonoscopy was performed using high-definition colonoscopy (Olympus 180, Tokyo, Japan). Although we did not use objective scoring for assessment of preparation, colonoscopy was only considered to be complete when mucosal visualization was considered complete in each segment after washing. Patients with inadequate preparation were rescheduled for colonoscopy. For dysplasia surveillance, we undertook fourquadrant random biopsies from each $10 \mathrm{~cm}$ interval during colonoscopy, including five random biopsies from the rectum. A minimum of 33 biopsies were therefore taken from each patient. Each biopsy was placed in separate vials and sent for histopathological analysis.

\section{Histopathological Assessment of Biopsy Specimens}

Two pathologists examined all the specimens for evidence of dysplasia separately in a blinded manner. The Vienna classification was used for grading of dysplasia, ${ }^{13}$ which grades the histology findings as: no dysplasia, indefinite for dysplasia, low-grade dysplasia, and high-grade dysplasia. The yield of dysplasia was then calculated.

\section{Statistical Analysis}

Descriptive statistics, i.e., the mean, SD, and frequency distribution were calculated for each variable in the study. Data were presented as a mean $\pm \mathrm{SD}$. The yield of dysplasia was then calculated by taking the total number of biopsies as the denominator. Statistical analysis was done using STATA for Windows version 12.1 . 


\section{RESULTS}

Between 2004 and December 2014, 1,693 patients with UC were registered at the IBD clinic at All India Institute of Medical Sciences. Diagnosis of UC was based on clinical history and associated endoscopic and histopathological findings. Among these patients, 136 underwent screening colonoscopy for CRC during the study period, which extended from March 2014 to July 2015. All of these patients had a duration of disease of more than 10 years and either left-sided colitis or pancolitis. Patients with a very high risk for developing dysplasia were included in this study and underwent random biopsies to detect dysplasia (Fig. 1).

Twenty-eight patients were included in this study. Twentysix of these patients had pancolitis and a duration of disease greater than 15 years, and two patients had PSC. None of the patients had a family history of CRC. The mean age of onset of disease in the study population was $28.89 \pm 8.73$ years, with a mean duration of disease of $19.00 \pm 8.78$ years. Eighteen patients (64.28\%) were males. The mean age of the study population at time of surveillance was $47.89 \pm 11.13$ years (Table 1). All patients were in clinical remission at the time of colonoscopy and had an SCCAI score of $<4$. All colonoscopies were performed by the same endoscopist, thus maintaining homogeneity across the study population.

At the time of colonoscopy, four patients had pseudopolyps. No other suspicious lesions were found during the procedure. A total of 924 random biopsies were taken, with at least 33 biopsies per patient. None of the biopsies had any evidence of definite dysplasia. Seven biopsies had features suggestive of "indefinite for dysplasia" (Fig. 2). The biopsies

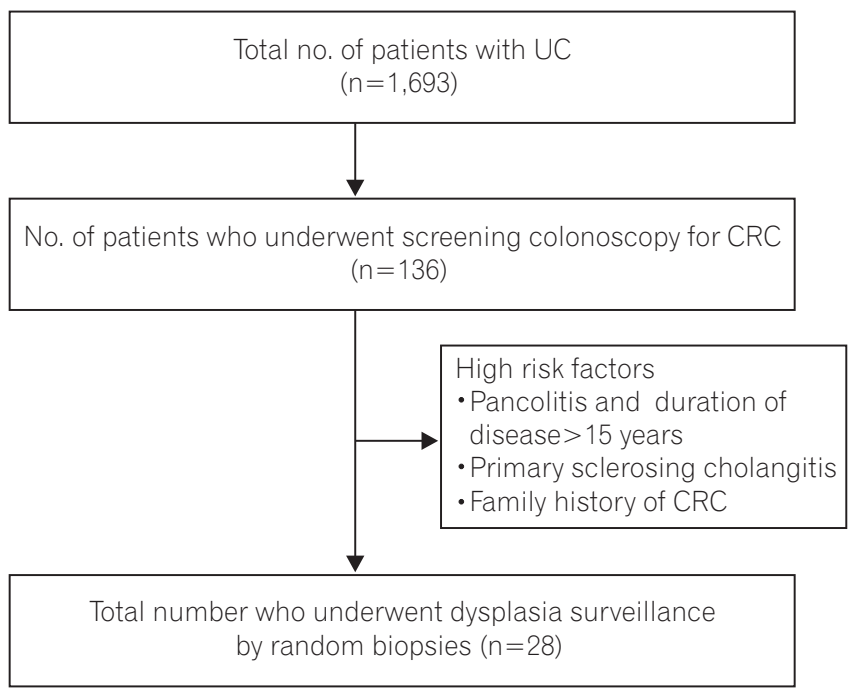

Fig. 1. Flow chart showing study design. CRC, colorectal cancer. in which colonic crypts showed nuclear stratification but maintained their elongated shape, with hyperchromasia and conspicuous nucleoli, in an area of neutrophilic cell infiltration or presence of inflammatory granulation tissue, were considered "indefinite for dysplasia." (Fig. 2)

\section{DISCUSSION}

Patients with UC have an increased risk of developing CRC. Screening for CRC and its precursor, dysplastic lesions, has universally been recommended in long-standing, extensive colitis. There is an increased risk of malignancy and dysplasia with increased duration of disease ${ }^{3}$ and increasing extent of disease. ${ }^{14}$ The other high-risk factors that predispose patients to developing malignancy include a family history of CRC and primary cholangitis. As our aim was to look at the effectiveness of random mucosal sampling for dysplasia, we included only patients with an expectedly high probability of dysplasia. Therefore, our study group excluded of patients with a disease duration of greater than 15 years and pancolitis, or a family history of CRC, or associated PSC (Fig. 1). The mean duration of disease was $19.00 \pm 8.78$ years. All of our patients had pancolitis, and two patients had PSC.

Early detection of dysplasia and thus precancerous lesions on colonoscopy in UC is challenging and difficult, for various reasons. Colitis-associated dysplasia, compared with sporadic adenoma or carcinoma, seems to have a distinct growth pattern, which can be flat and multifocal. ${ }^{15}$ Therefore, it is important that careful inspection of mucosa be undertaken during colonoscopy in high-risk patients for identification of these nonpolypoid areas of dysplasias. ${ }^{16}$ Traditionally, flat dysplasia in UC has been detected by random biopsies of

Table 1. Characteristics of Patients Who Underwent Screening for Dysplasia

\begin{tabular}{lc}
\hline \multicolumn{1}{c}{ Variable } & Random biopsy $(\mathbf{n = 2 8 )}$ \\
\hline Age at onset & $28.89 \pm 8.73$ \\
Duration of disease & $19.00 \pm 8.78$ \\
Age at time of surveillance & $47.89 \pm 11.13$ \\
Male gender & $18(64.28)$ \\
Endoscopic abnormalities & 0 \\
Total no. of biopsies & 924 \\
Dysplasia & \\
Indefinite for dysplasia & $7 / 924(0.70 \%)$ \\
Definite dysplasia & 0 \\
\hline
\end{tabular}

Values are presented as mean \pm SD or $n(\%)$. 

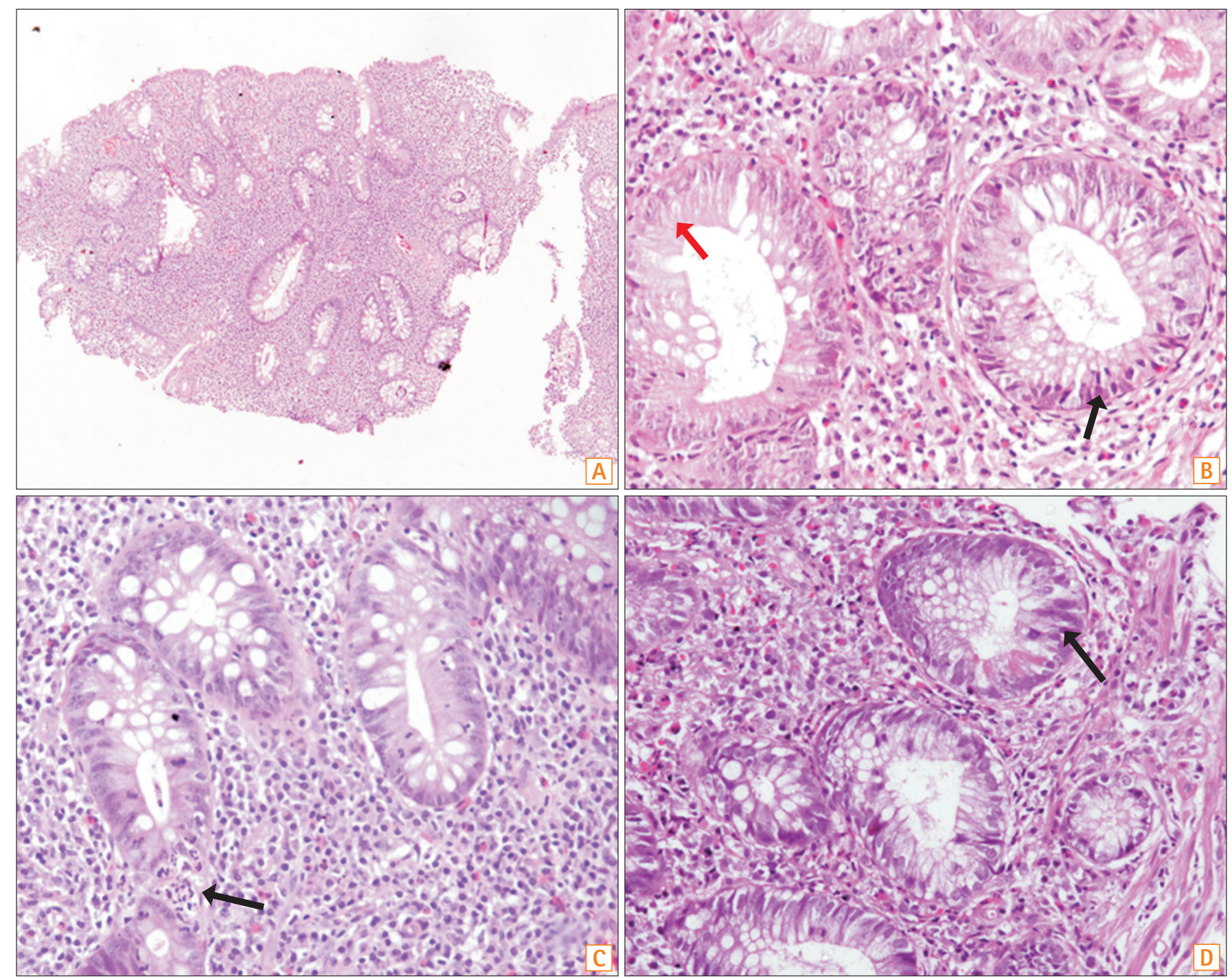

Fig. 2. Colonic biopsy specimens showing "Indefinite for dysplasia". Low-power photomicrograph shows colonic biopsy in ulcerative colitis with altered crypt architecture and dense mixed inflammation in lamina propria (A, H\&E x40). Focally, the crypt epithelial cells show nuclear stratification and hyperchromasia without prominent nucleoli, (B-D, HEE x400) in areas adjacent to neutrophilic infiltration (C, arrow), qualifying as indefinite for dysplasia changes.

mucosa that appeared unremarkable. ${ }^{17,18}$ However one study has shown that most of these flat dysplasias are endoscopically visible. ${ }^{19}$ In our study, though, we found no areas of abnormality or flat dysplasia on examination under white light. Thus, our study is similar to earlier studies where most raised lesions were not found by standard white light colonoscopy.

Chromoendoscopy-guided biopsies have been shown to be superior to random biopsies in detecting dysplasia in UC, with higher dysplasia yield in various large studies. ${ }^{9,10}$ Thus, various recent guidelines on screening for dysplasia recommend chromoendoscopy as the first choice for surveillance. ${ }^{8,20}$ The recent SCENIC (Surveillance for Colorectal
Endoscopic Neoplasia Detection and Management in Inflammatory Bowel Disease Patients: International Consensus Recommendations) guidelines ${ }^{20}$ have suggested that chromoendoscopy should be the standard of care, whereas the British Society of Gastroenterology endorses chromoendoscopy when available, but suggests that random biopsies can be undertaken when the expertise for chromoendoscopy is not available. In India, the training required to perform chromoendoscopy and recognize mucosal abnormalities is currently scarce. We therefore undertook this study to determine whether random biopsy would suffice for dysplasia detection until chromoendoscopy gains widespread 
popularity and is adopted by gastroenterologists throughout India. Furthermore, a few recent studies have shown that chromoendoscopy may not be superior to random biopsies. Mooiweer et al. ${ }^{21}$ retrospectively reviewed the results of 440 colonoscopies with chromoendoscopy and 1,802 colonoscopies using white light colonoscopy. Dysplasia was detected during 48 surveillance procedures (11\%) in the chromoendoscopy group compared with 189 procedures (10\%) in the white light endoscopy (WLE) group $(P=0.80)$. Targeted biopsies yielded 59 dysplastic lesions in the chromoendoscopy group, compared with the 211 dysplastic lesions detected in the WLE group $(P=0.30)$. Thus, the clinical impact of chromoendoscopy is still largely unknown.

In each patient in our study, we performed a minimum of 33 biopsies, which consisted of four-quadrant biopsies from each $10 \mathrm{~cm}$ interval during colonoscopy. A total of $924 \mathrm{bi}-$ opsies were examined, but none revealed definite dysplasia. Seven of the biopsies (from three patients) were labeled as indefinite for dysplasia. These changes were not interpreted as a true dysplasia, as such changes were not noted away from an area of active inflammation. Furthermore, nuclear rounding and nucleolar prominence were not as great as expected in dysplasia. It is to be highlighted that all the changes described in the "indefinite for dysplasia" cases were nuclear changes restricted to the basal half, and the outer glandular contour was maintained.

Random biopsies may not, therefore, suffice in the surveillance of dysplasia in patients with UC. We therefore conclude that a greater emphasis on training and familiarization of gastroenterologists with the technique of chromoendoscopy is currently needed, and is necessary for effective surveillance in long-standing UC.

\section{REFERENCES}

1. Ahuja V, Tandon RK. Inflammatory bowel disease: the Indian augury. Indian J Gastroenterol 2012;31:294-296.

2. Ahuja V, Tandon RK. Inflammatory bowel disease in the AsiaPacific area: a comparison with developed countries and regional differences. J Dig Dis 2010;11:134-147.

3. Eaden JA, Abrams KR, Mayberry JF. The risk of colorectal cancer in ulcerative colitis: a meta-analysis. Gut 2001;48:526-535.

4. Desai D, Shah S, Deshmukh A, et al. Colorectal cancers in ulcerative colitis from a low-prevalence area for colon cancer. World J Gastroenterol 2015;21:3644-3649.
5. Lutgens MW, Oldenburg B, Siersema PD, et al. Colonoscopic surveillance improves survival after colorectal cancer diagnosis in inflammatory bowel disease. Br J Cancer 2009;101:16711675.

6. Cairns SR, Scholefield JH, Steele RJ, et al. Guidelines for colorectal cancer screening and surveillance in moderate and high risk groups (update from 2002). Gut 2010;59:666-689.

7. Dignass A, Eliakim R, Magro F, et al. Second European evidence-based consensus on the diagnosis and management of ulcerative colitis part 1: definitions and diagnosis. J Crohns Colitis 2012;6:965-990.

8. Rubin CE, Haggitt RC, Burmer GC, et al. DNA aneuploidy in colonic biopsies predicts future development of dysplasia in ulcerative colitis. Gastroenterology 1992;103:1611-1620.

9. Kiesslich R, Fritsch J, Holtmann M, et al. Methylene blue-aided chromoendoscopy for the detection of intraepithelial neoplasia and colon cancer in ulcerative colitis. Gastroenterology 2003;124:880-888.

10. Kiesslich R, Neurath MF. Magnifying chromoendoscopy: effective diagnostic tool for screening colonoscopy. J Gastroenterol Hepatol 2007;22:1700-1701.

11. Picco MF, Pasha S, Leighton JA, et al. Procedure time and the determination of polypoid abnormalities with experience: implementation of a chromoendoscopy program for surveillance colonoscopy for ulcerative colitis. Inflamm Bowel Dis 2013;19:1913-1920.

12. Walmsley RS, Ayres RC, Pounder RE, Allan RN. A simple clinical colitis activity index. Gut 1998;43:29-32.

13. Schlemper RJ, Riddell RH, Kato Y, et al. The Vienna classification of gastrointestinal epithelial neoplasia. Gut 2000;47:251255.

14. Ekbom A, Helmick C, Zack M, Adami HO. Ulcerative colitis and colorectal cancer. A population-based study. N Engl J Med 1990;323:1228-1233.

15. Rutter M, Bernstein C, Matsumoto T, Kiesslich R, Neurath M. Endoscopic appearance of dysplasia in ulcerative colitis and the role of staining. Endoscopy 2004;36:1109-1114.

16. Leung K, Pinsky P, Laiyemo AO, Lanza E, Schatzkin A, Schoen RE. Ongoing colorectal cancer risk despite surveillance colonoscopy: the Polyp Prevention Trial Continued Follow-up Study. Gastrointest Endosc 2010;71:111-117.

17. Ueno Y, Tanaka S, Chayama K. Non-polypoid colorectal neoplasms in ulcerative colitis. Gastrointest Endosc Clin N Am 2010;20:525-542.

18. Sharan R, Schoen RE. Cancer in inflammatory bowel disease. An evidence-based analysis and guide for physicians and patients. Gastroenterol Clin North Am 2002;31:237-254. 
19. Rutter MD, Saunders BP, Wilkinson KH, Kamm MA, Williams $\mathrm{CB}$, Forbes A. Most dysplasia in ulcerative colitis is visible at colonoscopy. Gastrointest Endosc 2004;60:334-339.

20. Laine L, Kaltenbach T, Barkun A, et al. SCENIC international consensus statement on surveillance and management of dysplasia in inflammatory bowel disease. Gastrointest Endosc 2015;81:489-501.
21. Mooiweer E, van der Meulen-de Jong AE, Ponsioen CY, et al. Chromoendoscopy for surveillance in inflammatory bowel disease does not increase neoplasia detection compared with conventional colonoscopy with random biopsies: results from a large retrospective study. Am J Gastroenterol 2015;110:10141021. 